Robert W. Vallin, 229 Vincent Science Hall, Slippery Rock University of PA, Slippery Rock, PA 16057. e-mail: robert.vallin@sru.edu

\title{
CONTINUITY AND DIFFERENTIABILITY ASPECTS OF METRIC PRESERVING FUNCTIONS
}

\begin{abstract}
A function $f$ is metric preserving if for every metric space $(M, \rho)$ we have that $f \circ \rho$ is still a metric on $M$. In this article we look at the behavior of such functions with respect to continuity and differentiability. We include several pathological examples and some open questions.
\end{abstract}

\section{Introduction and Definitions}

It is well known (and can be found in many texts as a homework exercise) that any metric $d$ on any space $X$ can be turned into a bounded metric by composing $d$ with

$$
f(x)=\frac{x}{1+x} .
$$

This idea of retaining a metric under composition with some function $f$ is the motivation behind metric preserving functions.

Definition 1.1. Let $\mathbb{R}^{+}=[0, \infty)$. We call $f: \mathbb{R}^{+} \rightarrow \mathbb{R}^{+}$a metric preserving function if and only if $f \circ \rho: M \times M \rightarrow \mathbb{R}^{+}$is a metric for every metric $\rho$ where $(M, \rho)$ is an arbitrary metric space. Viewing these functions together as a set we let $\mathcal{M}$ denote the collection of metric preserving functions.

Obviously, any function of the form

$$
f(x)= \begin{cases}c & x>0 \\ 0 & x=0\end{cases}
$$

with $c>0$ will preserve metrics. There are also many interesting and nontrivial metric preserving functions to study. In [7] J. Doboš shows that the

Key Words: metric spaces, continuity, differentiability

Mathematical Reviews subject classification: Primary: 54E30 Secondary: 54E35, 26A21

Received by the editors February 23, 1999 
Cantor function is subadditive. Since a function which is subadditive, is zero at the origin, and is nondecreasing preserves metrics, the Cantor function is metric preserving.

The study of metric preserving functions goes at least as far back as [28] and preserving metrics has even appeared as a textbook exercise in [15]. The first in-depth discussion of $\mathcal{M}$ is in [21]. In this paper, we will mostly concentrate on these functions in terms of differentiability and continuity. We will also look at applications of these functions, and a few open questions. Proofs will not be emphasized. The interested reader will be referred to the appropriate source in the bibliography. However, we will provide most of the details on the construction of interesting and pathological examples.

Although we will restrict ourselves to functions from $\mathbb{R}^{+}$to $\mathbb{R}^{+}$we do note that there are papers on metric preserving functions of several variables ([19], [2], [3], [10] and [23]). They are defined on product spaces as follows: Let $T \neq \emptyset$ be a set of indices, and let $\left\{\left(M_{t}, d_{t}\right)\right\}_{t \in T}$ be a collection of metric spaces. We define $d:\left(\Pi_{t \in T} M_{t}\right)^{2} \rightarrow\left(\mathbb{R}^{+}\right)^{T}$ by

$$
d\left(\left(x_{t}\right)_{t \in T},\left(y_{t}\right)_{t \in T}\right)=\left(d\left(x_{t}, y_{t}\right)\right)_{t \in T} .
$$

Lastly we say $f:\left(\mathbb{R}^{+}\right)^{T} \rightarrow \mathbb{R}^{+}$is metric preserving if for every indexed collection of metric spaces $\left\{\left(M_{t}, d_{t}\right)\right\}_{t \in T}, f \circ d$ is a metric on $\left(\Pi_{t \in T} M_{t}\right)$.

\section{Some Basic Constructions and Properties}

We begin with some of the primary ways to create metric preserving functions. It is obvious that we must have $f(0)=0$ and there is no need to check for symmetry for $f \circ \rho$. Thus the only condition we must check is the triangle inequality. The following introductory constructions can be found in sources such as [1], [3] and [9].

Constructions Let $f: \mathbb{R}^{+} \rightarrow \mathbb{R}^{+}$.

1. If $f(a)=0 \Leftrightarrow a=0$, and $f$ is concave (down), then $f \in \mathcal{M}$.

2. If $f(0)=0$ and there exists an $a>0$ such that $a \leq f(x) \leq 2 a$ for all positive $x$, then $f \in \mathcal{M}$.

The latter is an especially effective means for creating examples. Given any function $F$ we can create the metric preserving $f$ via

$$
f(x)=\left\{\begin{array}{ll}
0 & x=0 \\
\frac{2}{\pi} \arctan (F(x))+1 & x>0
\end{array} .\right.
$$


We note here that this function is discontinuous at the origin although the original function $F(x)$ need not be, so some niceties can be lost using this idea.

Now that we have some established metric preserving functions, the following constructions allow us to create more. Methods 2 and 3 below are especially helpful in creating our later examples.

Constructions Let $f, g$, and the sequence $\left(f_{n}\right)$ be metric preserving functions.

1. The functions $f+g, \max \{f, g\}, f \circ g$, and $c \cdot f(c>0)$ are in $\mathcal{M}$.

2. If $\sum f_{n}$ converges to $f$, then $f \in \mathcal{M}$.

3. Let $\emptyset \neq \mathcal{L} \subseteq \mathcal{M}$. Suppose $\mathcal{L}_{x}=\{f(x): f \in \mathcal{L}\}$ is a bounded set for all $x>0$. Define $F$ by $F(x)=\sup \mathcal{L}_{x}$ for all $x \in \mathbb{R}^{+}$. Then $F \in \mathcal{M}$.

Instead of creating a function in $\mathcal{M}$, we also need to be able to see if a given function is metric preserving. This can be accomplished via the following analytic criteria.

Theorem 2.1. Let $f: \mathbb{R}^{+} \rightarrow \mathbb{R}^{+}$. Then $f \in \mathcal{M}$ if and only if

1. $f^{-1}(0)=\{0\}$, and

2. for all $a, b, c \geq 0,|a-b| \leq c \leq a+b$ implies $f(a) \leq f(b)+f(c)$.

A statement of this is found in [1], and a statement and proof can be found in [16] and [4].

As a consequence of meeting the triangle inequality $f$ must be subadditive (i.e. $f(a+b) \leq f(a)+f(b))$. However, as the following example shows, subadditivity is not enough.

Example 1. Define $f: \mathbb{R}^{+} \rightarrow \mathbb{R}^{+}$by

$$
f(x)= \begin{cases}x & 0 \leq x \leq 1 \\ 1 / 2 & 1<x\end{cases}
$$

This $f$ is subadditive, but not metric preserving.

Below are some of the properties for functions in $\mathcal{M}$ some of which are consequences of being metric preserving and subadditive.

Theorem 2.2. Let $f$ be a metric preserving function. Then

a. if $a \leq 2 b$ then $f(a) \leq 2 f(b)$, 
b. for all $a \geq 0$ and $n \in \mathbb{N}, 2^{-n} f(a) \leq f\left(2^{-n} a\right)$,

c. if $a \geq b$ then $f(a) / a \leq 2 \cdot f(b) / b$,

d. for all $a, b \in \mathbb{R}^{+},|f(a)-f(b)| \leq f(|a-b|)$.

We wish to end this section with some properties of functions in $\mathcal{M}$. The proofs for these are shown by using what we call triangle triplets. Triangle triplets first appeared in the literature in [23]. These triplets give a geometric description of metric preserving functions.

Definition 2.3. The triplet $(a, b, c)$ of real numbers is called a triangle triplet if and only if

$$
a \leq b+c, b \leq a+c, \text { and } c \leq a+b .
$$

Construction The function $f$ is in $\mathcal{M}$ if and only if $f$ vanishes exactly at zero and

$$
(f(a), f(b), f(c))
$$

is a triangle triplet whenever $(a, b, c)$ is one.

Once we have a collection of metric preserving functions, we can consider a sequence in $\mathcal{M}$ and whether or not this sequence converges (which, of course, depends on the metric for the set of functions). Even if we apply the supremum norm to the space of all functions than a convergent sequence of metric preserving functions need not be metric preserving. Just let

$$
f_{n}(x)=\left\{\begin{array}{ll}
1 / n & x>0 \\
0 & x=0
\end{array} .\right.
$$

So we ask the question when does a sequence in $\mathcal{M}$ converge in this norm to an element of $\mathcal{M}$ ?

Theorem 2.4. Let $\left\{f_{n}\right\}$ be a sequence of functions using the sup norm on the space of functions. If $f=\lim _{n \rightarrow \infty} f_{n}$ is positive for $x$ positive then $f$ is metric preserving.

Proof. Let $\varepsilon>0$ be arbitrary and $(a, b, c)$ be a triangle triplet. For this $\varepsilon$ there exists an $N$ such that $\left\|f_{n}-f\right\| \leq \varepsilon$ for all $n>N$. Then

$$
f(a) \leq f_{n}(a)+\varepsilon \leq f_{n}(b)+f_{n}(c)+\varepsilon .
$$

If we let $n$ approach infinity we have $f(a) \leq f(b)+f(c)+\varepsilon$ and since $\varepsilon$ is arbitrary (and the other cases are handled similarly) $(f(a), f(b), f(c))$ is a triangle triplet. Obviously $f(0)=0$ if and only if $x=0$, hence $f \in \mathcal{M}$. 
Note: With only slight changes in the proof we can relax to $f_{n}$ converging to $f$ pointwise.

Consider the following metric preserving $f$ :

$$
f(x)=\left\{\begin{array}{ll}
3 / 4 & x>0 \\
0 & x=0
\end{array} .\right.
$$

We wish to look at functions near $f$. However, we cannot use the open balls generated by the supremum norm to define "near" since it is obvious that not all $g$ in such a ball would be zero at the origin. But, if $g$ is a function from $\mathbb{R}^{+}$into $\mathbb{R}^{+}$and $g(0)=0$ then

$$
\sup _{x>0}|f(x)-g(x)|<1 / 4
$$

guarantees $g$ is also metric preserving. Keeping this in mind we give

Definition 2.5. Let $f: \mathbb{R}^{+} \rightarrow \mathbb{R}^{+}$be metric preserving. We define the open truncated ball of radius $\varepsilon$ around $f$ by

$$
\widehat{B}(f, \varepsilon)=\left\{g: \mathbb{R}^{+} \rightarrow \mathbb{R}^{+} \text {s.t. } g(0)=0 \text { and } \sup _{x>0}|f(x)-g(x)|<\varepsilon\right\} .
$$

Finally, we arrive at the question we wish to answer. Given the metric preserving $f$ under what condition on $f$ can we be assured there exists an $\varepsilon>0$ such that $\widehat{B}(f, \varepsilon) \subset \mathcal{M}$ ?

In order to answer this we must come up with a strengthening of the idea of a triangle triplet.

Definition 2.6. We say $f$ induces a sharp triangle triplet if there exists an $\eta>0$ such that for all triangle triplets $(a, b, c)$ with $a, b, c$ distinct we have

$$
\begin{aligned}
& f(a)+\eta \leq f(b)+f(c) \\
& f(b)+\eta \leq f(a)+f(c) \\
& f(c)+\eta \leq f(a)+f(b)
\end{aligned}
$$

The sharp triangle triplets are equivalent to saying there exists an $\alpha>0$ so that $f(x)>\alpha$ for all positive $x$. Now we have what we need to answer the question of functions near a metric preserving $f$.

Theorem 2.7. If $f \in \mathcal{M}$ induces a sharp triangle triplet then there exists an $\varepsilon>0$ such that $\widehat{B}(f, \varepsilon) \subset \mathcal{M}$. 
Proof. Let $\varepsilon=\eta / 3$ where $\eta$ comes from the definition of a sharp triangle triplet and let $g \in \widehat{B}(f, \varepsilon)$. Consider any triangle triplet $(a, b, c)$. Then

$$
g(a)<f(a)+\varepsilon \leq f(b)+f(c)-2 \varepsilon<g(b)+g(c) .
$$

Since the other cases are handled in a similar fashion we are done.

We wish to point out here that in these instances where such an $\varepsilon$ exists, the function $f$ cannot be continuous at the origin as then no such $\eta$ could be found to satisfy the sharp triangle triplet. Also we point out that this theorem is exact.

Example 2. If $f \in \mathcal{M}$, but does not induce a sharp triangle triplet then there is no $\varepsilon$ such that $\widehat{B}(f, \varepsilon) \subset \mathcal{M}$.

Proof. Since $f$ does not induce a sharp triplet, for the radius $\varepsilon / 2$ there exists a triangle triplet $(a, b, c)$ such that

$$
f(a)+\frac{\varepsilon}{2}>f(b)+f(c) .
$$

Pick a function $g \in \widehat{B}(f, \varepsilon)$ such that $g(a)=f(a)+\varepsilon / 2, g(b)=f(b)$ and $g(c)=f(c)$. This $g$ cannot be a metric preserving function.

\section{Continuity and Differentiability}

For a metric preserving function, the property of being continuous everywhere is a direct consequence of the function being continuous at the origin. From the fact that the image of $(h, x, x+h)$ is a triangle triplet we arrive at

$$
|f(x+h)-f(x)| \leq f(h)=f(h)-f(0) .
$$

Thus we conclude

Theorem 3.1. Let $f \in \mathcal{M}$. Then the following are equivalent:

1. $f$ is continuous,

2. $f$ is continuous at the origin,

3. for every $\varepsilon>0$ there exists an $x>0$ such that $f(x)<\varepsilon$.

Previously we have seen metric preserving functions that are continuous everywhere but $x=0$. The theorem above makes creating examples which are continuous a matter of making sure the example has continuity at the origin. 
There are many generalizations of continuity in the literature. We wish to point out that for many of these having this type of continuity at the origin will satisfy condition 3 above. Thus a metric preserving function having this generalized continuity at the origin would have ordinary continuity on $\mathbb{R}^{+}$. An example of this would be quasi-continuity. Other generalization (such as cliquishness) do not look directly at the point in question, but only "nearby." It is possible for a metric preserving function to be cliquish at the origin, but not continuous everywhere.

Let us insert here an interesting example of how badly some of the generalizations of continuity can be destroyed while creating a metric preserving function. This example comes from [6].

Example 3. Let $A$ and $B$ be sets which are disjoint while both are dense in $(0, \infty)$. Define $f$ by

$$
f(x)=\left\{\begin{array}{ll}
2 & x \in A \\
1 & x \in B \\
0 & x=0
\end{array} .\right.
$$

This function is metric preserving, but has bounded variation at no point.

However, P. Corazza in [19] used the notion of bounded gradient to relate metric preserving and bounded variation. For $r>0$ a metric preserving $f$ is of $r$-bounded gradient at 0 if there is a positive $h$ such that for all $x \in[0, h]$ we have $f(x) \leq r x$. We can than say the $f$ is of bounded gradient at 0 is for some positive $r$ we know $f$ is of $r$-bounded gradient.

Theorem 3.2. If $f$ is a metric preserving function that is of bounded gradient at 0 , then $f$ is of bounded variation.

Topologically, we have the following theorem concerning equivalent spaces. Proof of this can be found in [4].

Theorem 3.3. Let $(M, d)$ be a metric space. Let $f$ be a continuous metric preserving function. If $(M, d)$ is not discrete (not uniformly discrete), then the metrics $d$ and $f \circ d$ are equivalent (uniformly equivalent).

Moving on to differentiability, we see that once again behavior is tied to the behavior at the origin.

Theorem 3.4. Let $f \in \mathcal{M}$. Then $f^{\prime}(0)$ exists (even if $f^{\prime}(0)$ is not finite) and

$$
f^{\prime}(0)=\inf \left\{k>0: f(x) \leq k x \text { for all } x \in \mathbb{R}^{+}\right\} .
$$

The proof of this can be found in [1]. We can obtain even more if we know the derivative at zero is finite. Then we have 
Theorem 3.5. Let $f \in \mathcal{M}$ and $f^{\prime}(0)<\infty$. Then

1. for all $x \in \mathbb{R}^{+}, f(x)<k x$, and

2. for all $x, y \in \mathbb{R}^{+},|f(x)-f(y)| \leq f^{\prime}(0)|x-y|$.

We can rephrase the second part to say $f$ is Lipschitz with constant $f^{\prime}(0)$. Note that from Part 1 we have $f^{\prime}(0)<\infty$ equivalent to the bounded gradient. Also, we arrive at a corollary dealing with differentiability at points other than the origin.

Corollary 3.6. Let $f \in \mathcal{M}$ be differentiable. Then $\left|f^{\prime}(x)\right| \leq f^{\prime}(0)$ for each $x \in \mathbb{R}^{+}$.

Interesting examples concerning metric preserving functions and differentiation are already known (see [11], [25], and [9]). We present a few of these here, but we start with two more ways to create functions in $\mathcal{M}$. The triangle triplets defined in the previous section are used in proofs that the following constructions do indeed lead to functions in $\mathcal{M}$.

Construction Let $f \in \mathcal{M}$. Let $d, k>0$ such that $g(x)=k x$ for $x \in[0, d)$ and $g(x)=f(x)$ on $[d, \infty)$. Then $g \in \mathcal{M}$ if and only if

1. $f(d)=k d$, and

2. for all $x, y \in[d, \infty)$

$$
|f(x)-f(y)| \leq k|x-y| .
$$

Using this we come up with (see [11])

Example 4. There exists a metric preserving function which is continuous and nowhere differentiable.

Proof. We now start with the metric preserving $g$ where $g(x)$ is given by

$$
g(x)= \begin{cases}0 & x=0 \\ \frac{1}{2}+\left|x-[x]+\frac{1}{2}\right| & x>0\end{cases}
$$

with $[x]$ being the integer part of $x$. Then the function $f$ given by

$$
f(x)= \begin{cases}x & 0 \leq x \leq 1 \\ g(x) & 1<x\end{cases}
$$


is metric preserving using the construction above. Finally, we have the metric preserving

$$
F(x)=\sum 2^{-n} f\left(2^{n} x\right)
$$

which is continuous and nowhere differentiable.

The idea used above was then modified by Doboš and Z. Piotrowski in [11] to the next construction. This will be used to construct a function where the derivative exists everywhere and is infinite at certain points.

Construction Let $g, h \in \mathcal{M}$. Let $d>0$ such that $g(d)=h(d)$. Define $w: \mathbb{R}^{+} \rightarrow \mathbb{R}^{+}$by

$$
w(x)=\left\{\begin{array}{ll}
g(x) & 0 \leq x<d \\
h(x) & d \leq x<\infty
\end{array} .\right.
$$

Suppose $g$ is nondecreasing and concave and satisfies

$$
\text { for all } x, y \in[d, \infty) \text { with }|x-y| \leq d,|h(x)-h(y)| \leq g(|x-y|) .
$$

Then $w \in \mathcal{M}$.

Example 5. There exists a continuous metric preserving function $f$ which is everywhere differentiable (in the extended sense) and $f^{\prime}(x)=\infty$ for all $x$ of the form $2^{-n}, n \in \mathbb{N}$.

ProOF. Now we start with the function $g(x)$ where

$$
g(x)=\left\{\begin{array}{ll}
\sqrt{2 x-x^{2}} & 0 \leq x \leq 1 \\
1 & 1<x
\end{array} .\right.
$$

It is easy to see that this is in $\mathcal{M}$ because $g$ is concave down and nondecreasing. Next, we use $g$ to define $h$ by

$$
h(x)=\left\{\begin{array}{ll}
0 & x=0 \\
1 & 0<x<1 \\
\frac{1}{2}(3-g(2-x)) & 1 \leq x<2 \\
\frac{1}{2}(3+g(x-2)) & 2 \leq x
\end{array} .\right.
$$

For $x \neq 0,1 \leq h(x) \leq 2$, hence $h$ preserves metrics. Putting $g$ and $h$ together using the last construction we arrive at the metric preserving $w: \mathbb{R}^{+} \rightarrow \mathbb{R}^{+}$ given by

$$
w(x)=\left\{\begin{array}{ll}
g(x) & 0 \leq x<1 \\
h(x) & 1 \leq x<\infty
\end{array} .\right.
$$


This $w$ has the following properties:

$$
\begin{aligned}
& w \text { is continuous and nondecreasing } \\
& w \text { is bounded } \\
& w^{\prime}(x) \text { exists (in the extended sense) for all } x \geq 0 \\
& w^{\prime}(2)=\infty
\end{aligned}
$$

Finally, let

$$
f(x)=\sum 2^{-n} w\left(2^{n} x\right) .
$$

After this result, Doboš and Piotrowski asked if characterizing $\left\{x: f^{\prime}(x)=\right.$ $\infty\}$ was possible. Since

$$
\left|f^{\prime}(x)\right| \leq f^{\prime}(0),
$$

if the set in question is nonempty, it must contain the origin. A partial answer to this was given in [25].

Example 6. Let $Z$ be a measure zero $\mathcal{G}_{\delta}$ set. There exists a continuous metric preserving function whose derivative exists everywhere and is infinite precisely on $Z \cup\{0\}$.

Proof. As noted in Lemma 1.2, Chapter 2 of [5] given this set $Z$ there exists an absolutely continuous $G(x)$ such that

$$
G^{\prime}(x)=\infty \text { for } x \in Z \text { and } G^{\prime}(x) \text { exists and is finite for } x \notin Z \text {. }
$$

Define $\widehat{G}(x)=\frac{2}{\pi} \arctan (G(x))+1$. This $\widehat{G}(x)$ is clearly uniformly continuous.

Let $s(x)$ be a continuous increasing differentiable concave function that maps $\mathbb{R}^{+}$onto $\mathbb{R}^{+}$such that $s(0)=0$ and for all $h \in(0,2)$

$$
s(h) \geq 2 \sup \left\{|\widehat{G}(x+h)-\widehat{G}(x)|: x \in \mathbb{R}^{+}\right\} .
$$

Using this $s$ we can put together a sequence of continuous, differentiable metric preserving functions $f_{m}$.

Start with $\left\{a_{m}\right\}$, a sequence of points in $(0,1) \backslash Z$ converging monotonically to zero. For each $m$ find the point $b_{m}$ such that $s\left(b_{m}\right)=\widehat{G}\left(a_{m}\right)$. Since for all but finitely many $m$ the condition $b_{m}>\frac{1}{2} a_{m}$ must be true we require that it is true for all $m$ in our sequence. Define

$$
f_{m}(x)= \begin{cases}s\left(\left(2 b_{m} x\right) / a_{m}\right) & x \in\left[0, a_{m} / 2\right] \\ t(x) & x \in\left[a_{m} / 2, a_{m}\right] \\ \widehat{G}(x) & x \in\left[a_{m}, \infty\right)\end{cases}
$$

where $t(x)$ 
1. is a differentiable function with $1 \leq t(x) \leq 2$,

2. meets $t\left(a_{m} / 2\right)=s\left(b_{m}\right), t\left(a_{m}\right)=\widehat{G}\left(a_{m}\right)$, and $t$ connects $s$ and $\widehat{G}$ smoothly, and

3. satisfies $|t(x)-t(y)| \leq \frac{1}{2} s\left(\frac{2 b_{m}}{a_{m}}|x-y|\right)$ for $|x-y| \leq a_{m} / 2$.

Now $s\left(\left(2 b_{m} x\right) / a_{m}\right)$ is metric preserving since it's increasing, concave and $s(0)=0$ and the function

$$
y= \begin{cases}0 & x=0 \\ t\left(a_{m} / 2\right) & x \in\left(0, a_{m} / 2\right] \\ t(x) & x \in\left[a_{m} / 2, a_{m}\right] \\ \widehat{G}(x) & x \in\left[a_{m}, \infty\right)\end{cases}
$$

is metric preserving since $1 \leq y \leq 2$ for $x>0$. Putting $s$ and $y$ together shows $f_{m}$ is metric preserving. Note that $f_{m}^{\prime}(x)=\infty$ on $\left(\left[a_{m}, \infty\right) \cap Z\right) \cup\{0\}$ and elsewhere $f_{m}^{\prime}$ exists and is finite. Lastly, let

$$
f(x)=\sum 2^{-m} f_{m}(x) .
$$

As previously stated, $f^{-1}(0)=\{0\}$ and $f$ concave will ensure $f$ is metric preserving. It is easy to see that if $f$ is strictly convex on some interval $[0, s]$ that it cannot be in $\mathcal{M}$. As the following example shows, this does not mean $f$ cannot be convex in many intervals near 0 .

Example 7. [1] There is a function $f \in \mathcal{M}$ such that

1. $f$ is differentiable,

2. $f^{\prime}$ is continuous on $(0, \infty)$,

3. $f$ is not increasing on any neighborhood of 0 ,

4. each neighborhood of zero contains an interval on which $f$ is strictly convex.

Proof. For each natural number $n$, let $r_{n}=\frac{(2 n-1)(n+1)}{(2 n+1) n^{2}}$ and let

$$
g_{n}(x)= \begin{cases}0 & x=0 \\ a_{n} x^{3}+b_{n} x^{2}+c_{n} x+d_{n} & x \in\left[r_{n}, 1 / n\right) \\ \left(2-\frac{1}{n}\right) \frac{1}{n} & \text { otherwise }\end{cases}
$$


where

$$
\begin{array}{r}
a_{n}=\left(16 n^{7}+24 n^{6}+8 n^{5}-2 n^{4}-n^{3}\right)(n+1)^{-1}, \\
b_{n}=\left(-48 n^{6}-72 n^{5}-12 n^{4}+18 n^{3}+2 n^{2}-2 n\right)(n+1)^{-1} \\
c_{n}=\left(48 n^{6}+72 n^{5}-30 n^{3}+n^{2}+5 n-1\right)\left(n^{2}+1\right)^{-1} \\
d_{n}=\left(-16 n^{4}-8 n^{3}+12 n^{2}+2 n-2\right) n^{-1} .
\end{array}
$$

For each $g_{n}$ there exists a positive constant $k_{n}$ so that $k_{n} \leq g_{n}(x) \leq 2 k_{n}$ for $x>0$. Hence each $g_{n}$ is metric preserving. Next, let

$$
f_{n}(x)= \begin{cases}\left(2-\frac{1}{n+1}\right) x & x \in\left[0, r_{n}\right) \\ g_{n}(x) & x \in\left[r_{n}, \infty\right)\end{cases}
$$

and define $f_{0}(x)=x$. Each $f_{n}$ is metric preserving and the function we seek is

$$
F(x)=\sup _{n}\left\{f_{n}(x)\right\} .
$$

If $f$ is differentiable, then convexity can be thought of as having $f^{\prime}$ increase. F. Terpe, in [23], shows any increase in $f^{\prime}$ cannot continue without bound.

Theorem 3.7. If $f: \mathbb{R}^{+} \rightarrow \mathbb{R}^{+}$is differentiable on some neighborhood of $\infty$ and $\lim _{x \rightarrow \infty} f^{\prime}(x)=\infty$, then $f$ is not metric preserving.

As this last example shows, if we replace lim with limsup in the theorem above then we can reach a different conclusion.

Example 8. [1] There is a function $f \in \mathcal{M}$ such that

1. $f$ is continuous,

2. $f^{\prime}(0)=\infty$,

3. $f$ is differentiable on $(0, \infty)$,

4. $\lim \sup _{x \rightarrow \infty} f^{\prime}(x)=\infty$

Proof. For each $i \in \mathbb{N}$, let $a_{i}=1-\sqrt{1-2^{-2 i}}$. Define $h_{i}$ by

$$
h_{i}(x)=\left\{\begin{array}{ll}
0 & x=0 \\
2^{-i-1} & x \in\left(0, a_{i+1}\right) \\
2^{-i-2}\left(3+\sin \frac{\pi\left(2 x-a_{i}-a_{i+1}\right)}{2\left(a_{i}-a_{i+1}\right)}\right) & x \in\left[a_{i+1}, a_{i}\right) \\
2^{-i} & x \in\left(a_{i}, \infty\right)
\end{array} .\right.
$$


Each $h_{i}$ is metric preserving since $x>0$ implies $2^{-i-1} \leq h(x) \leq 2^{-i}$. Now let

$$
g_{i}(x)=\left\{\begin{array}{ll}
\left(2^{i+1} a_{i+1}\right)^{-1} x & x \in\left[0, a_{i}\right) \\
h_{i}(x) & x \in\left[a_{i}, \infty\right)
\end{array} .\right.
$$

Because $\left|h_{i}^{\prime}(x)\right| \leq\left(2^{i+1} a_{i+1}\right)^{-1}$ we have $g_{i} \in \mathcal{M}$. Define the metric preserving function $t_{n}$ by

$$
t_{n}(x)=\sup _{i \geq n} g_{i}(x)
$$

Now for each $i$ let

$$
r_{i}(x)= \begin{cases}0 & x=0 \\ 2^{-i-1}\left(3+\cos \frac{2(x-i-1)}{a_{i}}\right) & x \in\left[i+1-\frac{\pi}{4} a_{i}, 1+i+\frac{\pi}{4} a_{i}\right] . \\ 2^{-i} & \text { otherwise }\end{cases}
$$

Each $r_{i}$ is metric preserving since for nonzero $x, 2^{-i} \leq r_{i}(x) \leq 2^{-i+1}$. If we let

$$
s_{i}(x)= \begin{cases}\left(2^{i} a_{i}\right)^{-1} x & x \in\left[0, a_{i}\right) \\ r_{i}(x) & x \in\left(a_{i}, \infty\right)\end{cases}
$$

then $s_{i} \in \mathcal{M}$ because $\left|s_{i}^{\prime}(x)\right| \leq\left(2^{i} a_{i}\right)^{-1}$. Lastly, define

$$
f_{0}(x)= \begin{cases}\sqrt{2 x-x^{2}} & x \in[0,1) \\ 1 & x \in[1, \infty)\end{cases}
$$

and for each $n$ let $f_{n}(x)=\max \left\{t_{n}(x), s_{n}(x)\right\}$. All $f_{n}$ are metric preserving and now the example we want is $\sum f_{n}$.

\section{Applications for Functions in $\mathcal{M}$}

As previously stated, metric preserving functions can be used to guarantee a space is bounded. M. Jůza, in 1956 in [14], gave the first application for nonmonotone metric preserving functions. He was dealing with the following situation: If $P$ is a complete metric space, it is known that given $K_{n}$ a sequence of closed balls such that $K_{n+1} \subseteq K_{n}$ and the radii of the balls is converging to zero then $\cap K_{n} \neq \emptyset$. The example below shows this is not true if we leave out the requirement that the radii converge to zero.

Example 9. Let $P=\mathbb{N}$, the set of natural numbers with metric $\rho(m, m)=0$ and if $m \neq n$

$$
\rho(m, n)=1+\frac{1}{\min \{m, n\}} .
$$

Then letting $K_{n}$ be the closed ball about $b$ of radius $1+1 / n$ will suffice. 
Jůza, however, considered this insufficient since the space in the example consists of isolated points. In his paper he constructs a metric on $\mathbb{R}$ so that the space contains a sequence of closed balls with empty intersection. To begin with, he shows that with the function given by

$$
f(x)= \begin{cases}x & x \leq 2 \\ 1+\frac{1}{x-1} & x>2\end{cases}
$$

$f(|x-y|)$ is a metric on $\mathbb{R}$ and that $\mathbb{R}$ is complete with this. Lastly induction is used to define the $K_{n}$.

The proof of Jůza's result is based on ensuring the property that for all compact sets $K$ there exists a closed set $S$ and a compact set $L$ such that

$$
K \subseteq \mathbb{R}-S \subseteq L
$$

In [12] Doboš and Piotrowski looked more closely at this condition. They developed the next theorem while searching for conditions than ensure $(\mathrm{J})$.

Theorem 4.1. Let $f \in \mathcal{M}$ and $g, h: \mathbb{R} \rightarrow \mathbb{R}$ such that $g$ and $h$ are nonincreasing and nonconstant in each neighborhood of $\infty$ and

$$
g(x) \leq f(x) \leq h(x)
$$

in some neighborhood of $\infty$ and $\lim _{x \rightarrow \infty} g(x)=\lim _{x \rightarrow \infty} h(x)$. Then $(\mathbb{R}, f \circ|\cdot|)$ has $(J)$.

However, as the next example shows, in order for a function to satisfy $(\mathrm{J})$ it need not satisfy the condition of Doboš and Piotrowski's theorem. The $f$ below satisfies the condition while being monotone in no neighborhood of infinity.

Example 10. [12] Define $f: \mathbb{R}^{+} \rightarrow \mathbb{R}^{+}$as

$$
f(x)=\left\{\begin{array}{ll}
x & 0 \leq x<1 \\
\frac{1+x+\sin ^{2}(x-1)}{2 x} & 1 \leq x
\end{array} .\right.
$$

It remains an open question to characterize the metric preserving functions $f$ such that $(\mathbb{R}, f \circ|\cdot|)$ is a complete metric space containing a monotone sequence of closed balls with empty intersection.

Let us note here that satisfying $(\mathrm{J})$ is not necessary to have a monotone sequence with empty intersection. This example also comes from [12]. 
Example 11. Define $f: \mathbb{R}^{+} \rightarrow \mathbb{R}^{+}$by the following: On $[0,1]$ we have $f(x)=x$. On $(3 n-2,3 n+1], n=1,2,3, \ldots$ let

$$
\begin{aligned}
f(x)=\frac{1}{2}(x-3 n+1-|x-3 n+1| & +\left|x-3 n+\frac{n+4}{2(n+2)}\right| \\
& \left.+\left|x-3 n-\frac{n+4}{2(n+2)}\right|\right)
\end{aligned}
$$

This $f$ satisfies the sequence of closed balls condition without satisfying (J).

Another application of metric preserving functions was remarked upon by S. Watson in [27]. We know that nondecreasing subadditive functions are metric preserving. The scalings used in statistics are nondecreasing and thus we have "a complete description of scaling functions which work for any set of distance data."

\section{Subsets of Metric Preserving Functions}

Along with the collection of metric preserving functions, subsets of the set $\mathcal{M}$ have been studied in papers (see [26] and [20]). The first dealt with differentiation and metric preserving functions. The second paper looked at periodic functions in $\mathcal{M}$.

Definition 5.1. Let $f \in \mathcal{M}$ be differentiable with finite derivative on $(0, \infty)$. Define $g(x)$ as

$$
g(x)=\left\{\begin{array}{ll}
f^{\prime}(x) & x>0 \\
0 & x=0
\end{array} .\right.
$$

We say $f \in \mathcal{D}$ if and only if $g \in \mathcal{M}$.

Some of the results from this paper include

Theorem 5.2. If $f$ is differentiable on $[0, \infty)$ and metric preserving then $f^{\prime}$ is not a metric preserving function.

Theorem 5.3. If $f \in \mathcal{D}$, then $f$ is nondecreasing.

Theorem 5.4. Let $f(x)=x^{k}$. Then $f \in D$ if and only if $k=1$.

Finally, this paper gives a way to construct functions in $D$. 
Construction Let $g: \mathbb{R}^{+} \rightarrow \mathbb{R}^{+}$be a function satisfying

$$
\forall a>0 \quad \int_{0}^{a} g(x) d x \geq \int_{b}^{c} g(x) d x \text { where } c-b=a .
$$

Let $N$ and $M$ be real numbers. If there exists an $A>0$ such that

$$
A \leq N+M g(x) \leq 2 A,
$$

then both $G(x)=\left\{\begin{array}{ll}N+M g(x) & x>0 \\ 0 & x=0\end{array}\right.$ and $F(x)=\int_{0}^{x} G(t) d t$ are in $\mathcal{M}$.

This leads to another, new, way to create function in $\mathcal{M}$.

Construction Let $g: \mathbb{R}^{+} \rightarrow \mathbb{R}^{+}$be an integrable function satisfying for all $a>0$

$$
\int_{0}^{a} g d \lambda \geq \int_{b}^{c} g d \lambda
$$

where $c-b=a$ and $\lambda$ denotes Lebesgue measure. Then $\int_{0}^{x} g d \lambda \in \mathcal{M}$.

This last construction is similar to one in [23]. There the author stated that if $g: \mathbb{R}^{+} \rightarrow \mathbb{R}^{+}$is decreasing, continuous and positive-valued then

$$
f(x)=\int_{0}^{x} g(x) d x
$$

is metric preserving.

The second subset we wish to look at was investigated by I. Pokorný in [20]. He concerned himself with functions in $\mathcal{M}$ which are related to periodic functions. Obviously, no metric preserving function can be periodic (since $f(x)=0$ if and only if $x=0$ ). Instead we will look at the set of $f \in \mathcal{M}$ where $f(x)=x+g(x)$ and $g$ is periodic. Two examples of these functions are

$$
f(x)=x+|\sin x|
$$

and

$$
F(x)=[x]+\sqrt{x-[x]}
$$

where [.] is the greatest integer function. In his paper, Pokorný gave the following characterization of these functions.

Theorem 5.5. Let $f(x)=x+g(x)$ where $g$ is nonconstant and periodic. Then $f \in \mathcal{M}$ if and only if the following conditions hold: 
- $\forall a \in \mathbb{R}^{+}, f(a)=0$ if and only if $a=0$,

- $f$ is subadditive, and

- $f$ is nondecreasing.

\section{Preserving the Euclidean Metric}

Recently some work has been done on preserving not all metric spaces $(M, \rho)$, but just the real line with the Euclidean metric ([8] and [16]). Obviously, $\mathcal{M}$ is a subset of this collection. As we shall see, it is a proper subset and this larger set contains some very interesting examples. The test to determine whether or not a function preserves the Euclidean metric can again be based on triangle triplets.

Construction The function $f: \mathbb{R}^{+} \rightarrow \mathbb{R}^{+}$preserves the Euclidean metric on $\mathbb{R}$ if and only if $f(0)=0$ and $f$ maps $(a, b, a+b)$ into a triangle triplet.

Denote the space of all function which preserve $(\mathbb{R},|\cdot|)$ as $\mathcal{M}^{*}$. Obviously $\mathcal{M} \subset \mathcal{M}^{*}$. An example that shows the inclusion is proper is the following from $[16]$ :

$$
f(x)=\left\{\begin{array}{ll}
x & x \text { is rational } \\
1+x & x \text { is not rational }
\end{array} .\right.
$$

A continuous example in $\mathcal{M}^{*}$, but not $\mathcal{M}$, was given by Doboš in [8] as

$$
g(x)=|\sin x|+|\sin \sqrt{2} x| .
$$

Note how different this is from metric preserving functions. In this example $\liminf _{x \rightarrow \infty} g(x)=0$. For $f \in \mathcal{M}$ it is known that for all $a, b \geq 0$

$$
a \leq 2 b \text { implies } f(a) \leq 2 f(b) .
$$

Hence as $x$ approaches infinity there is some $c>0$ which the function cannot go below.

In [8] there is a construction which shows how to create a function which preserves the Euclidean metric, but where $\liminf _{x \rightarrow \infty} f(x)=0$. We begin with some lemmas.

Lemma. Let $f$ be a metric preserving function. For each natural number $n$ define $f_{n}: \mathbb{R}^{+} \rightarrow \mathbb{R}^{+}$by

$$
f_{n}(x)= \begin{cases}f(x) & x \in\left[0,2^{n-1}\right] \\ f\left(2^{n}-x\right) & x \in\left(2^{n-1}, 2^{n}\right] \\ f_{n}\left(x-k \cdot 2^{n}\right) & x \in\left(k \cdot 2^{n},(k+1) 2^{n}\right], k \in \mathbb{N} .\end{cases}
$$


Then $f \circ|\cdot|$ is a pseudometric on $\mathbb{R}$.

Construction Let $f$ be a metric preserving function. Suppose that $f(x)=1$ for $x \geq 1$. Define $f_{0}: \mathbb{R}^{+} \rightarrow \mathbb{R}^{+}$by

$$
f_{0}(x)=\sup _{n \in \mathbb{N}}\left\{2^{-n} \cdot f_{n}(x)\right\} .
$$

Then $f \circ|\cdot|$ is a metric on the real line and $f_{0}\left(2^{n}\right)=2^{-n}$ for each $n \in \mathbb{N}$.

Doboš goes even further. He constructs a function which preserves the Euclidean metric, has $\liminf _{x \rightarrow \infty} f(x)=0$, and is differentiable.

Example 12. For all natural numbers, define the values $a_{n}$ and $k_{n}$ by

$$
a_{n}=\frac{n+1}{n \cdot 2^{n}} \text { and } k_{n}=\frac{2^{1-n}}{a_{n}} .
$$

Let $f: \mathbb{R}^{+} \rightarrow \mathbb{R}^{+}$have

a. $f(0)=0$,

b. $f$ is nondecreasing,

c. $f$ is differentiable on $[0, \infty)$,

d. $f\left(a_{n}\right)=2^{1-n}$,

e. $f^{\prime}\left(a_{n}\right)=0$,

f. $f(x)=1$ for $x \geq 1$,

g. $f(x) \geq k_{n} x$ for each $x \in\left(a_{n+1}, a_{n}\right)$, and

h. $f^{\prime}(x) \leq k_{n+1}$ for each $x \in\left(a_{n+1}, a_{n}\right)$.

Then $f$ is metric preserving since $f(x)=\sup _{n} g_{n}(x)$ where

$$
g_{n}(x)= \begin{cases}k_{n+1} x & x \in\left[0, a_{n+1}\right) \\ f(x) & x \in\left[a_{n+1}, a_{n}\right] . \\ 2^{1-n} & x \in\left(a_{n}, \infty\right)\end{cases}
$$

Using the above construction we can create $f_{0}(x)$ which meets all of our goals.

It's then stated that if the $f$ is continuous then $f_{0}$ is almost periodic. The question is then asked

Is every continuous $f \in \mathcal{M}^{*}$ with $\liminf _{x \rightarrow \infty} f(x)=0$ almost periodic?

This question has recently been answered in the negative by this author [24]. 


\section{References}

[1] Borsík, J. and Doboš, J., On metric preserving functions, Real Analysis Exchange, 13(1987-88), 285-293.

[2] Borsík, J. and Doboš, J., On metrization of the uniformity of a product of metric spaces, Math. Slovaca, 32(1982), 97-102.

[3] Borsík, J. and Doboš, J., On a product of metric spaces, Math. Slovaca, 31(1981), 193-205.

[4] Borsík, J. and Doboš, J., Functions whose composition with every metric is a metric, Math. Slovaca, 31(1981), 3-12 (in Russian).

[5] Bruckner, A., Differentiation of real functions, 2nd Ed., Rhode Island, AMS, 1994.

[6] Corazza, Paul, Introduction to metric-preserving functions, Amer. Math. Monthly, 106(4), 309-323.

[7] Doboš, J., The standard Cantor function is subadditive, Proc. Amer. Math. Soc., 124(1996), 3425-3426.

[8] Doboš, J., On modifications of the Euclidean metric on the reals, Tatra Mountains Math. Publ., 8(1996), 51-54.

[9] Doboš, J., A survey of metric preserving functions, Questions and Answers in General Topology, Vol 13(1995), 129-134.

[10] Doboš, J., On a certain lattice of topologies on a product of metric spaces, Math. Slovaca, 32(1982), 397-402.

[11] Doboš, J. and Piotrowski, Z., Some remarks on metric preserving functions, Real Analysis Exchange, 19(1993-1994), 317-320.

[12] Doboš, J. and Piotrowski, Z., A note on metric preserving functions, Inter. J. Math and Math. Sci., 19(1996), 199-200.

[13] Doboš, J. and Piotrowski, Z., When distance means money, Int. J. Math. Educ. Sci. Technol., 28(1997), 513-518.

[14] Jůza, M., A note on complete metric spaces, Matematicko-Fyzikálny Časopis SAV, VI, 3(1956), 143-148 (Czech).

[15] Kelley, J.L., General Topology, Van Nostrand, New York, 1955. 
[16] Law, M., "Absolutely" metric preserving functions: functions that preserve the absolute value metric, Master's Project, the University of North Dakota, 1995.

[17] Piotrowski, Z., On integer-valued metrics, School of Math., Phys. Chem. Wroclaw Univ. (Poland), 1974 (Polish).

[18] Pokorný, I., Remarks on the sum of metrics, Tatra Mountains Math. Publ., 14(1998), 63-65.

[19] Pokorný, I., Some remarks on metric preserving functions of several variables, Tatra Mountains Math. Publ., Vol 8(1996), 89-92.

[20] Pokorný, I., Some remarks on metric preserving functions, Tatra Mountains Math. Publ., 2(1993), 66-68.

[21] Sreenivasan, T.K., Some properties of distance functions, J. Indian Math. Soc., 11(1947), 38-43.

[22] Terpe, F., Some properties of metric preserving functions, Proc. Conf. Topology, Measure, and Fractals, Math. Res. 66, Akademie-Verlag, Berlin, (1992), 214-217.

[23] Terpe, F., Metric preserving functions of several variables, Proc. Conf. Topology and Measure V, Greifswald (1988), 169-174.

[24] Vallin, R.W., On preserving ( $\mathbb{R}$, Eucl.) and almost periodic functions, (submitted).

[25] Vallin, R.W., On metric preserving functions and infinite derivatives, Acta Math. Univ. Comenianae, Vol 67, No.2(1998), 373-376.

[26] Vallin, R.W., A subset of metric preserving functions, Int. J. Math. and Math. Sci., Vol. 21, No. 2, 409-410.

[27] Watson, S., Review of A survey of metric preserving functions, Mathematical Reviews, AMS, $96 \mathrm{~m}$.

[28] Wilson, W.A., On certain types of continuous transformations of metric spaces, Amer. J. Math. 57(1935), 62-68. 